Review Article

\title{
Managing Aggressive Behaviors among Psychiatric Inpatients Through the Use of Restrictive Methods
}

\author{
Anas Husam Khalifeh \\ Department of Nursing, Prince Hamzah Hospital, Ministry of Health of the Hashemite Kingdom of Jordan, Amman, Jordan \\ Email address: \\ anaskhalifeh@yahoo.com

\section{To cite this article:} \\ Anas Husam Khalifeh. Managing Aggressive Behaviors Among Psychiatric Inpatients Through the Use of Restrictive Methods. American \\ Journal of Psychiatry and Neuroscience. Vol. 4, No. 5, 2016, pp. 71-75. doi: 10.11648/j.ajpn.20160405.11
}

Received: July 22, 2016; Accepted: August 10, 2016; Published: August 25, 2016

\begin{abstract}
The most aggressive behaviors were common in patients who diagnosed with schizophrenia, major depressive disorder, bipolar disorder, and substance use disorders. The prevalence in Jordan $23.6 \%$ of psychiatric inpatients occurred with them aggressive behavior. There are interventions and ways to control the aggressive behaviors include seclusion, physical restraints, time out, and chemical restraints. The aim of this paper is to highlight on the concepts about aggressive behaviors, seclusion, physical restraints and factors that consider using seclusion and physical restraints and the effective methods were used with aggressive behaviors among psychiatric inpatients. Searching strategies used an online database that searched include PubMed, Wiley, EBSCO host, Ovid database, and Google Scholar from 2006 to January 2016. The most articles of physical restraints and seclusion that use among psychiatric inpatients setting work on to reduce it and to know factors of aggressive behaviors to move away from using it. Recommendation train staff nurse to managing with aggressive behaviors, work as multidisciplinary staff, and creating a therapeutic environment.
\end{abstract}

Keywords: Restraints, Psychiatric Inpatients, Chemical Restraints, Physical Restraints, Seclusion, Aggressive Behaviors

\section{Introduction}

Aggressive behavior is the behavior that harms other or self physically or emotionally, that may be physical or verbal aggression behaviors [1], although; should be interventions that be effective and safe to prevent injury to everyone involved [2], this behavior cause severe complication during treatment [3]. However, that prevalence of aggressive behavior happened in about 30\% with psychiatric patients who admitted to the first time in the mental health center [4]. Particularly in Jordan, as in [5] reported $23.6 \%$ of psychiatric inpatients had aggressive behavior.

Moreover, reference [6] conducted aggressive behaviors which recorded by psychiatric nurses includes: verbal aggressive behavior (96.2\%), aggressive behavior on others $(84 \%)$, aggressive behavior on certain objects $(79.7 \%)$ and self-injury $(77.7 \%)$. There is an important clinical duty to prevent aggressive behavior which by identifies to risk factors to occurs this behavior [3]. Aggressive behaviors most common in patients who diagnosed with schizophrenia [7], major depressive disorder, bipolar disorder, and substance use disorders [8]. There are interventions and ways to control the aggressive behaviors include seclusion, physical restraints, time out, and chemical restraints [9].

Physical restraints is a historically way and common practice use as psychiatric intervention, mechanical restraints during the 17th and early 18 th centuries were seen as a necessary in asylum life [10]. Although, all definition concepts of physical restraints mean almost the same meaning and including, intervention that keeps patients in bed and uses cotton belt fixing the patients to limit the patient's physical mobility [2], and defined as webbing a patient to a bed with mechanical devices (belts). This is a bed with belts over the patient's arms, legs, and trunk [11].

Seclusion is means isolating psychiatric inpatients in locked rooms that prepared specially and safely separate from other patients, used internationally in wide shape to manage disturbed behavior from psychiatric inpatients $[12,13]$ and the same thing in seclusion have more than one definition but all means the same, seclusion also involves isolation and the 
reduction of sensory stimuli [14].

There are reasons for isolate psychiatric inpatients in seclusion include; violence to property, verbal aggression or threats, threats of self-harm or actual self-harm, physical aggression to others, and severe psychiatric symptoms or disturbed behavior [13]. However, there is a lack of prevalence of seclusion and physical restraints [15], although many studies investigated the intervention, the methods of calculation and reported prevalence rates vary widely [16].

Based on articles that searched to covered the aim of this paper is to highlight on the concepts about aggressive behaviors, seclusion, physical restraints and the relationship to each other and factors that consider using seclusion and physical restraints and the effective methods was used with aggressive behaviors among psychiatric inpatients. The question is what are effective methods seclusion or physical restraints in aggressive behaviors among psychiatric inpatients?

\section{Search Strategies}

Depending on the evidence-based practice way to answer this question by used online database that searched include PubMed, Wiley, EBSCO host, Ovid database, and Google Scholar from 2006 to January 2016 with the following combination of keywords: restraints, psychiatric inpatients, chemical restraints, physical restraints, seclusion, aggressive behaviors.

\section{Literature Review}

Most of the articles discussed to reduce the physical restraints and seclusion among psychiatric inpatients for more than reason, although there are some of the debates about this topic [17], however; will view this topic and look at more than one point view. However, according to American Psychiatric Association (APA) suggested indications for use of restraint and seclusion: prevention harm to self and others, prevention of damage to the physical environment, prevention of serious disruption of the treatment program, a contingency in the behavior therapy of dangerous behaviors, decrease of stimulation, and the patient's request [18]. Otherwise; the differences in legal supply make differences of use the physical restraint and seclusion, showed by Netherlands has a high rate of restraint and aggression in Europe related to the restrictive use of involuntary medication. In the UK mechanical restraints are prohibited with the only physical holding of a patient upright or on the floor for less than half an hour. In Canada their 2-year retrospective study conducted that $23.2 \%$ were secluded with or without restraints and $17.5 \%$ were secluded with restraints [19].

\subsection{Physical Restraint}

Physical restraints also have important implications for nurses and other staff and defined previously as webbing a patient to a bed with mechanical devices (belts) [11]. Whereas, physical restraints as a necessary intervention that from the perspective of some nurse and professionals, the percentage of psychiatric inpatients demonstrate aggressive behavior was $16 \%$ of during the first week of hospitalization and $7 \%$ of persons with a mental disorder have perpetrated aggression in the year after their diagnosis, compared with $2 \%$ in the general population [20]. However, physical restraints still used in a psychiatric setting for safety regardless of the disadvantages [21].

\subsection{Seclusion}

Seclusion used to manage risk and disturbed behavior on psychiatric wards, can't eliminated completely from psychiatric units a still topics for discussion [13], also as in [12] reported that some psychiatric hospitals in the UK work without use seclusion on their psychiatric setting whether in acute psychiatric ward or psychiatric intensive care units. Although; there are several psychiatric hospitals in several countries have a system about large level action to reduce seclusion use, however, hospitals in the UK use to be a low usage of seclusion [13]. Beside that many studies showed this topic as dilemmas to use it with aggressive psychiatric patients [17].

References [13, 22] explained that seclusion more likely used with physical aggressive, also aggressive behavior may repetition during patient in the hospital when used seclusion more than used other methods and seclusion wasn't acceptable for the patient and staff nurse. In addition, the use of seclusion that linked with the availability of seclusion room that increase rate use of seclusion, and that wasn't shown any connection with reducing aggressive behavior, self-harm, and medication-related conflict $[23,24]$.

In Jordan, the consideration of 20 Jordanian nurses worked in mental health services about seclusion it is a treatment and necessary for aggressive patients. The nurses had a lack of knowledge and practice to deal with aggressive patients and did not have the clear connotation of seclusion [25]. On the other hand, there is a policy about the use of seclusion from the Jordanian nursing council used by National Center for Mental Health (NCMH), which considered guideline of action for all medical staff [26].

\subsection{Factors to Use Physical Restraint and Seclusion}

There are factors that considered from nurse to make a decision for seclusion or restraints or other methods that include personal characteristics of patients, healthcare team, and environment, explained by the personal characteristics of patients that the men are more likely to physical restraints [27, 28], seclusion more likely with the young and the middle-aged patients [29] and restraints too [21], patients who suffering from schizophrenia or bipolar disease are more likely to seclusion or restraints [29, 30], on the other hand; the informal norms that define the position of healthcare team factor that relation to decision of secluding or restraining patients, environment factor that reflect by level of education of staff and how manage with aggressive patients and the environmental area should be calm to release the anxiety $[29,31]$.

Moreover, some of the personal characteristics of patients not reasonable to alter with seclusion [32]. Also, increase the 
length of stay in psychiatric hospital associated with frequency of physical restrained [28], stay greater than 6 days in psychiatric hospital higher risk of being restrained, increased number of admissions ( 3 or more), and younger age (18-29 years) [21].

\subsection{Reduce Using for Physical Restraint and Seclusion}

The practice of secluding in psychiatric setting have an argument and are high-risk practice [33], so there is program built upon the Public Health Prevention Model call Crisis Prevention Management that focuses on changing the culture of patient care, by changing the philosophy of care to reduce the usage of seclusion and restraints [34]. Moreover; the success interventions to reduce seclusion or restraints include: mobilization of staff and daily stimulation sessions, observation and team meetings regarding patients considered at high risk of a seclusion, individual support for team members with stress laid on their needs, reorganization of the environment: rehabilitation structure and consideration of staff culture, coaching, and group support [35].

On the other hand; in [36] reported that to reducing physical restraints by shorten the length of stay in the psychiatric setting and decrease possibility to readmission include active and assertive treatment after discharge, furthermore; use active and assertive treatment pre and post discharge help in that and by use milieu treatment [37]. In addition; [38] suggested reducing restraints by identification of potential aggression, adequate drug treatment, and personal interventions. However, physical restraints have restricted the freedom of patient and incompatible with the principle of autonomy [39].

Furthermore, these procedures are associated with negative physical consequences for patients including lacerations, asphyxiation, and even death [40]. The other reasons that call to reduce restraints, [41] found that psychiatric inpatients who're restrained have an incidence of deep vein thrombosis $11.6 \%$ even with the use of unfractionated heparin and long duration of restraints, excessive sedation and lower antipsychotic medication associated with increased incidence of deep vein thrombosis. However, de-escalation technique used as an alternative intervention, [42] in the acute psychiatric inpatient setting used de-escalation as aggression management is the first-line intervention and conducted de-escalation was successful in approximately $60 \%$ of cases. De-escalation improves safety during practice for both staff and patients [43].

\section{Conclusion and Recommendation}

The most literature review of physical restraints and seclusion that use among psychiatric inpatients setting work on to reduce it and to know factors of aggressive behaviors to move away from using it, in addition, there is a lack of studies defend of physical restraints and seclusion. The author opinion is the use of these methods in mental health hospital are more than normal as punishment and there is studies implication to manage to use these methods for aggressive behaviors which effect on patient's life and may threaten and high-risk procedures. Ways to control the aggressive behaviors include seclusion, physical restraints, time out, and chemical restraints. On the other hand, physical restraints and seclusion used in psychiatric setting significantly and for many reasons included fear of staff, low level of qualification, self-harm, harm to other and aggressive behaviors, however; there are other methods to manage with these reasons without using physical restraints and seclusion like de-escalation techniques, so should reduce use physical restraints and seclusion.

In addition, there are characteristics or factors that play an important role in nurse decision to response on aggressive behaviors which can show nurse how can work with patients out of the use of physical restraints and seclusion. The priority in mental health hospitals is safety, no effective treatment without safety. Finally, decommissioning of seclusion rooms remains impossible which conclusion on whether would be the best way to minimize or eliminate seclusion use and physical restraints.

The author recommended how to manage aggressive behaviors to training staff nurse to deal with aggressive behaviors and to know when and how the use of coercive interventions, which might reduce recourse to seclusion and restraints, and work for multidisciplinary staff in restraining process. Furthermore, give staff nurse program of clinical supervision to assist in manage distressing emotions and creating a therapeutic environment that may help to use these interventions by reducing the behaviors that effect on patient oneself and others.

However, most articles have shown weakness by the lack of evidence-based knowledge about the physical restraints and seclusion if they have benefit or harm effect and lack of data to assess the real use of these methods. In addition, some studies do not allow to collect information from patient's record, the policy of some countries is different so they affect to collect data and generalize the result, and this data from the nursing note that not accurate. Conversely, the strength of some studies collected data from the large sample and take a long time on studies.

\section{References}

[1] Gabbey, A. (2013). Aggressive Behavior. Healthline. Retrieved 13 May 2016, from http://www.healthline.com/health/aggressive-behavior\#Overvi ew1

[2] Huf, G., Coutinho, E., Ferreira, M., Ferreira, S., Mello, F., \& Adams, C. (2011). TREC-SAVE: a randomised trial comparing mechanical restraints with use of seclusion for aggressive or violent seriously mentally ill people: study protocol for a randomised controlled trial. Trials, 12 (1). doi: $10.1186 / 1745-6215-12-180$

[3] Ketelsen, R., Zechert, C., Driessen, M., \& Schulz, M. (2007). Characteristics of aggression in a German psychiatric hospital and predictors of patients at risk. J Psychiatr Ment Health Nurs, 14 (1). doi: 10.1111/j.1365-2850.2007.01049.x 
[4] Dean, K., Walsh, E., Morgan, C., Demjaha, A., Dazzan, P., \& Morgan, K. et al. (2006). Aggressive behaviour at first contact with services: findings from the AESOP First Episode Psychosis Study. Psychological Medicine, 37 (04), 547-557. doi: $10.1017 / \mathrm{s} 0033291706008920$

[5] Al-Sagarat, A., Hamdan-Mansour, A., Al-Sarayreh, F., Nawafleh, H., \& Moxham, L. (2015). Prevalence of aggressive behaviours among inpatients with psychiatric disorders: A case study analysis from Jordan. Nursing \& Health Sciences, 18 (2), 172-179. doi: 10.1111/nhs.12239

[6] Kim, Y. O. (2010). Analysis of nursing intervention according to type of aggressive behavior in psychiatric inpatients. Unpublished master's thesis, Ewha Womans University, Seoul

[7] Amoo, G., \& Fatoye, F. O. (2010). Aggressive behaviour and mental illness: a study of in-patients at Aro Neuropsychiatric Hospital, Abeokuta. Nigerian journal of clinical practice, 13 (3), 351-355

[8] Friedman, R. (2006). Violence and Mental Illness - How Strong is the Link? New England Journal of Medicine, 355(20), 2064-2066. doi: 10.1056/nejmp068229

[9] Migon, M., Coutinho, E., Huf, G., Adams, C., Cunha, G., \& Allen, M. (2008). Factors associated with the use of physical restraints for agitated patients in psychiatric emergency rooms. General Hospital Psychiatry, 30 (3), 263-268. doi: 10.1016/j.genhosppsych.2007.12.005

[10] Carr, P. (2012). The use of mechanical restraint in mental health: a catalyst for change?.Journal of Psychiatric and Mental Health Nursing, 19(7), 657-664. doi: 10.1111/j.1365-2850.2012.01912.x

[11] Husum, T., Bjørngaard, J., Finset, A., \& Ruud, T. (2010). A cross-sectional prospective study of seclusion, restraint and involuntary medication in acute psychiatric wards: patient, staff and ward characteristics. BMC Health Services Research, 10 (1). doi: 10.1186/1472-6963-10-89

[12] Bowers, L., Van Der Merwe, M., Nijman, H., Hamilton, B., Noorthorn, E., Stewart, D., \& Muir-Cochrane, E. (2010). The Practice of Seclusion and Time-out on English Acute Psychiatric Wards: The City-128 Study. Archives of Psychiatric Nursing, 24 (4), 275-286. doi: 10.1016/j.apnu.2009.09.003

[13] Bowers, L., Ross, J., Nijman, H., Muir-Cochrane, E., Noorthoorn, E., \& Stewart, D. (2011). The scope for replacing seclusion with time out in acute inpatient psychiatry in England. Journal of Advanced Nursing, 68 (4), 826-835. doi: 10.1111/j.1365-2648.2011.05784.x

[14] Mayers, P., Keet, N., Winkler, G., \& Flisher, A. (2010). Mental Health Service Users' Perceptions and Experiences of Sedation, Seclusion and Restraint. International Journal of Social Psychiatry, 56 (1), 60-73. doi: $10.1177 / 0020764008098293$

[15] Stewart, D., Van der Merwe, M., Bowers, L., Simpson, A., \& Jones, J. (2010). A Review of Interventions to Reduce Mechanical Restraint and Seclusion among Adult Psychiatric Inpatients. Issues in Mental Health Nursing, 31 (6), 413-424. doi: $10.3109 / 01612840903484113$

[16] Janssen, W., Noorthoorn, E., de Vries, W., Hutschemeakers, G., Lendemeijer, H., \& Widdershoven, G. (2008). The use of seclusion in the Netherlands compared to countries in and outside Europe. International Journal of Law And Psychiatry, 31 (6), 463-470. doi: 10.1016/j.ijlp.2008.09.002

[17] Khalifeh, A. (2015). Position Statement: The Use of Seclusion in Psychiatric Settings. Middle East Journal of Nursing, 9 (3), 31-36. doi: 10.5742/mejn.2015.92662

[18] American Psychiatric Association (APA). (2006). The Use of Restraint and Seclusion in Correctional Mental Health Care. http://www.psychiatry.org/File\%20Library/Learn/Archives /rd2006_Seclusion.pdf: American Psychiatric Association

[19] Dumais, A., Larue, C., Drapeau, A., Ménard, G., \& Giguére Allard, M. (2011). Prevalence and correlates of seclusion with or without restraint in a Canadian psychiatric hospital: a 2-year retrospective audit. Journal of Psychiatric and Mental Health Nursing, 18 (5), 394-402. doi: 10.1111/j.1365-2850.2010.01679.x

[20] Choe, J., Teplin, L., \& Abram, K. (2008). Perpetration of Violence, Violent Victimization, and Severe Mental Illness: Balancing Public Health Concerns. PS, 59 (2), 153-164. doi: 10.1176/ps.2008.59.2.153

[21] Knutzen, M., Bjørkly, S., Eidhammer, G., Lorentzen, S., Helen Mjøsund, N., \& Opjordsmoen, S. et al. (2013). Mechanical and pharmacological restraints in acute psychiatric wards - Why and how are they used? Psychiatry Research, 209 (1), 91-97. doi: 10.1016/j.psychres.2012.11.017

[22] Whittington, R., Bowers, L., Nolan, P., Simpson, A., \& Neil, L. (2009). Approval Ratings of Inpatient Coercive Interventions in a National Sample of Mental Health Service Users and Staff in England. Psychiatric Services, 60 (6). doi: 10.1176/appi.ps.60.6.792

[23] Bowers, L., Allan, T., Simpson, A., Jones, J., Van Der Merwe, M., \& Jeffery, D. (2009). Identifying Key Factors Associated with Aggression on Acute Inpatient Psychiatric Wards. Issues in Mental Health Nursing, 30 (4), 260-271. doi: $10.1080 / 01612840802710829$

[24] Baker, J., Bowers, L., \& Owiti, J. (2009). Wards features associated with high rates of medication refusal by patients: a large multi-centred survey. General Hospital Psychiatry, 31 (1), 80-89. doi: 10.1016/j.genhosppsych.2008.09.005

[25] Khudhur, I. (2013). Nurses' knowledge about psychiatric patient seclusion in Jordan. Kufa Journal for Nursing Sciences, $3(3)$

[26] National Center for Mental Health. (2011). Policies and Procedures for Psychiatric Nursing. Jordan: Jordanian nursing council, 1-165

[27] Hendryx, M., Trusevich, Y., Coyle, F., Short, R., \& Roll, J. (2009). The Distribution and Frequency of Seclusion and/or Restraint among Psychiatric Inpatients. The Journal of Behavioral Health Services \& Research, 37 (2), 272-281. doi: 10.1007/s11414-009-9191-1

[28] Knutzen, M. (2011). Characteristics of Psychiatric Inpatients Who Experienced Restraint and Those Who Did Not: A Case-Control Study. Psychiatric Services, 62 (5), 492. doi: 10.1176/appi.ps.62.5.492

[29] Larue, C., Dumais, A., Ahern, E., Bernheim, E., \& Mailhot, M (2009). Factors influencing decisions on seclusion and restraint. Journal of Psychiatric and Mental Health Nursing, 16 (5), 440-446. doi: 10.1111/j.1365-2850.2009.01396.x 
[30] Hammer, J., Springer, J., Beck, N., Menditto, A., \& Coleman, J. (2010). The Relationship Between Seclusion and Restraint Use and Childhood Abuse Among Psychiatric Inpatients. Journal of Interpersonal Violence, 26 (3), 567-579. doi: $10.1177 / 0886260510363419$

[31] Downey, L., Zun, L., \& Gonzales, S. (2007). Frequency of alternative to restraints and seclusion and uses of agitation reduction techniques in the emergency department. General Hospital Psychiatry, 29 (6), 470-474. doi: 10.1016/j.genhosppsych.2007.07.006

[32] Vruwink, F., Noorthoorn, E., Nijman, H., VanDerNagel, J., Hox, J., \& Mulder, C. (2012). Determinants of Seclusion After Aggression in Psychiatric Inpatients. Archives of Psychiatric Nursing, 26 (4), 307-315. doi:10.1016/j.apnu.2011.10.004

[33] Khalifeh, A. (2015). Arguments of Legal and Ethical Use of Seclusion among Psychiatric Inpatients. Middle East Journal of Psychiatry and Alzheimers, 6 (2), 12-20. doi: 10.5742/mepa.2015.92730

[34] Lewis, M., Taylor, K., \& Parks, J. (2009). Crisis Prevention Management: A Program to Reduce the Use of Seclusion and Restraint in an Inpatient Mental Health Setting. Issues in Mental Health Nursing, 30 (3), 159-164. doi: $10.1080 / 01612840802694171$

[35] Sclafani, M., Humphrey II, F., Repko, S., Ko, H., Wallen, M., \& DiGiacomo, A. (2007). Reducing Patient Restraints: A Pilot Approach Using Clinical Case Review. Perspectives in Psychiatric Care, 44 (1), 32-39. doi: 10.1111/j.1744-6163.2008.00145.x

[36] Zhang, J., Harvey, C., \& Andrew, C. (2011). Factors associated with length of stay and the risk of readmission in an acute psychiatric inpatient facility: a retrospective study. Aust NZ J Psychiatry, 45 (7), 578-585. doi: $10.3109 / 00048674.2011 .585452$
[37] Vaaler, A., Iversen, V., Morken, G., Fløvig, J., Palmstierna, T., \& Linaker, O. (2011). Short-term prediction of threatening and violent behaviour in an Acute Psychiatric Intensive Care Unit based on patient and environment characteristics. BMC Psychiatry, 11(1). doi: 10.1186/1471-244x-11-44

[38] Gelkopf, M., Roffe, Z., Behrbalk, P., Melamed, Y., Werbloff, N., \& Bleich, A. (2009). Attitudes, Opinions, Behaviors, and Emotions of the Nursing Staff Toward Patient Restraint. Issues in Mental Health Nursing, 30 (12), 758-763. doi: $10.3109 / 01612840903159777$

[39] Mohr, W. (2010). Restraints and the code of ethics: An uneasy fit. Archives of Psychiatric Nursing, 24 (1), 3-14. doi: 10.1016/j.apnu.2009.03.003

[40] Paterson, B. \& Duxbury, J. (2007). Restraint and the Question of Validity. Nursing Ethics, 14 (4), 535-545. doi: $10.1177 / 0969733007077888$

[41] Ishida, T., Katagiri, T., Uchida, H., Takeuchi, H., Sakurai, H., Watanabe, K., \& Mimura, M. (2014). Incidence of Deep Vein Thrombosis in Restrained Psychiatric Patients.

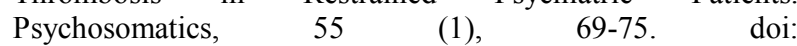
10.1016/j.psym.2013.04.001

[42] Lavelle, M., Stewart, D., James, K., Richardson, M., Renwick, L., Brennan, G., \& Bowers, L. (2016). Predictors of effective de-escalation in acute inpatient psychiatric settings. J Clin Nurs, 25 (15-16), 2180-2188. doi: 10.1111/jocn.13239

[43] Price, O., Baker, J., Bee, P., \& Lovell, K. (2015). Learning and performance outcomes of mental health staff training in de-escalation techniques for the management of violence and aggression. The British Journal of Psychiatry, 206 (6), 447-455. doi: 10.1192/bjp.bp.114.144576 